\title{
Fourier Integral Operators Defined by Classical Symbols with Exit Behaviour
}

\author{
Sandro Coriasco ${ }^{* \dagger}$ and Paolo Panarese $e^{\ddagger}$ \\ Dipartimento di Matematica \\ Università di Torino \\ V. C. Alberto, n. 10, \\ I-10123, Torino, ITALY
}

\begin{abstract}
We continue the investigation of the calculus of Fourier Integral Operators (FIOs) in the class of symbols with exit behaviour ( $\mathbf{S G}$ symbols). Here we analyse what happens when one restricts the choice of amplitude and phase functions to the subclass of the classical SG symbols. It turns out that the main composition theorem, obtained in the environment of general SG classes, has a "classical" counterpart. As an application, we study the Cauchy problem for classical hyperbolic operators of order $(1,1)$; for such operators we refine the known results about the analogous problem for general SG hyperbolic operators. The material contained here will be used in a forthcoming paper to obtain a Weyl formula for a class of operators defined on manifolds with cylindrical ends, improving the results obtained in [9].
\end{abstract}

\section{Introduction}

The calculus of FIOs developed in [4] is based on the class of (general) SG symbols and amplitudes, i.e., the classes of all $a \in \mathcal{C}^{\infty}\left(\mathbb{R}^{n} \times \mathbb{R}^{n} \times \mathbb{R}^{n} ; \mathbb{C}\right)$ satisfying $\forall \alpha, \beta, \gamma \in \mathbb{N}^{n} \exists C_{\alpha \beta \gamma} \geq 0$ such that

$$
\left|\partial_{\xi}^{\alpha} \partial_{\beta}^{x} \partial_{\gamma}^{y} a(x, y, \xi)\right| \leq C_{\alpha \beta \gamma}\langle\xi\rangle^{m_{1}-|\alpha|}\langle x\rangle^{m_{2}-|\beta|}\langle y\rangle^{m_{3}-|\gamma|}
$$

where, as usual, $|\alpha|=\alpha_{1}+\alpha_{2}+\ldots+\alpha_{n}$ for all $\alpha \in \mathbb{N}^{n},\langle x\rangle=\sqrt{1+|x|^{2}}$ for all $x \in \mathbb{R}^{n}$ and $(x, y, \xi)$ runs through all $\mathbb{R}^{n} \times \mathbb{R}^{n} \times \mathbb{R}^{n}$. When (1.1) is fulfilled, we say that $a$ belongs to the class of $\mathbf{S G}$ amplitudes of order $m=\left(m_{1}, m_{2}, m_{3}\right)$. An SG (left) symbol is an SG amplitude which does not depend on $y$. In such a case, the vanishing third component of the order is dropped, and we will use

\footnotetext{
*Supported by the EU Research and Training Network "Geometric Analysis".

$\dagger$ E-mail: Coriasco@dm.unito.it

†E-mail: Panarese@dm.unito.it
} 
the double order $m=\left(m_{1}, m_{2}\right)$, denoting the set of $\mathbf{S G}$ symbols by $\mathbf{S G}_{l}^{m}$. With some requirement on the real-valued phase function $\varphi \in \mathbf{S G}_{l}^{(1,1)}$ (see below), it turns out that FIOs of the type

$$
A u(x)=A_{\varphi, a} u(x)=\frac{1}{(2 \pi)^{n}} \int e^{i \varphi(x, \xi)} a(x, \xi) \hat{u}(\xi) d \xi
$$

make sense for $u \in \mathcal{S}\left(\mathbb{R}^{n}\right), a \in \mathbf{S G}_{l}^{m}, m \in \mathbb{R}^{2}$. More precisely, the operator in (1.2) (Type I FIO) is linear and continuous from $\mathcal{S}\left(\mathbb{R}^{n}\right)$ to itself and extendable to a linear continuous operator from $\mathcal{S}^{\prime}\left(\mathbb{R}^{n}\right)$ to itself. All these results hold for Type II FIOs as well, i.e. the operators of the form

$$
\widehat{B u}(\xi)=\widehat{B_{\varphi, b}} u(\xi)=\int e^{-i \varphi(x, \xi)} \overline{b(x, \xi)} u(x) d x .
$$

These and other results (in particular, about the compositions of this FIOs with the corresponding SG pseudo-differential operators ( $\psi$ dos $)$ ) were obtained in [4], and subsequently applied to the study of SG-hyperbolic Cauchy problems in [5] and [7]. In section 2 we fix some notations and recall the essentials from the SG $\psi$ dos calculus (see Cordes [2], Cordes and Erkip [3], Schrohe [11, 12], Schrohe and Erkip [13] for details and further development of the theory), together with a short resume of the calculus of the SG FIOs, developed in [4]. In the same section we define classical SG symbols, introduced in Egorov and Schulze [8] and Schulze [14] (see also Maniccia and Panarese [9]). We will denote the subclass of $\mathbf{S G}$ classical (left) symbols of order $m \in \mathbb{R}^{2}$ by $\mathbf{S G}_{l, c l}^{m}$. Here we wish to illustrate what happens when amplitude and phase functions of SG FIOs (1.2) and (1.3) are classical SG symbols ${ }^{1}$. In such a case, we will briefly speak of classical SG FIOs. It is possible to prove the following theorem.

Theorem 1.1 Let $P=\mathrm{Op}(p)$ be a classical $\psi$ do and let $A=A_{\varphi, a}$ be a classical FIO of Type I with $p \in \mathbf{S G}_{l, c l}^{r}, a \in \mathbf{S G}_{l, c l}^{s}$. Then, the composed operators $P A$ and $A P$ are classical FIOs with the same phase function and amplitude $h$ such that $h \in \mathbf{S G}_{l, c l}^{r+s}$. The same holds for PB and BP, where $B$ is a classical FIO of Type II with amplitude function $b \in \mathbf{S G}_{l, c l}^{s}$.

Section 3 is devoted to the precise definition of classical FIOs and to the proof of Theorem 1.1 above, while in section 4 we will discuss the hyperbolic first order Cauchy problem associated with classical SG operators, following the same lines of [5]. We will prove the following theorem.

Theorem 1.2 Assume that $\lambda$ is a classical SG symbol of order $(1,1)$ depending smoothly on a parameter $t \in J=[-T, T], T>0$. Assume also that the corresponding operator is hyperbolic ${ }^{2}$. Then, the operator $A_{\varphi(t), a(t)}$, approximating the solution operator of

$$
\left\{\begin{array}{l}
\left(D_{t}-\Lambda(t)\right) u(t)=0, \quad t \in J \\
u(0)=u_{0}
\end{array}\right.
$$

modulo smoothing operators, is a classical SG FIO.

\footnotetext{
${ }^{1}$ The additional requirements for the phase function will be stated in section 3 .

${ }^{2}$ See below for the precise definitions.
} 


\section{SG classes of symbols and operators. SG Sobolev spaces}

We set, from now on,

$$
\begin{aligned}
d_{x} \varphi & =\left(\frac{\partial \varphi}{\partial x^{1}}, \ldots, \frac{\partial \varphi}{\partial x^{n}}\right)=\left(\partial_{1} \varphi, \ldots, \partial_{n} \varphi\right)=\left(\partial_{1}^{x} \varphi, \ldots, \partial_{n}^{x} \varphi\right) \\
\nabla_{\xi} \varphi & =\left(\begin{array}{c}
\frac{\partial \varphi}{\partial \xi_{1}} \\
\ldots \\
\frac{\partial \varphi}{\partial \xi_{n}}
\end{array}\right)=\left(\begin{array}{c}
\partial^{1} \varphi \\
\ldots \\
\partial^{n} \varphi
\end{array}\right)=\left(\begin{array}{c}
\partial_{\xi}^{1} \varphi \\
\ldots \\
\partial_{\xi}^{n} \varphi
\end{array}\right) .
\end{aligned}
$$

When there is no reference to asymptotic expansions, the notations $f \sim g$ and $f(x) \sim g(x)$ will mean $\exists C_{1}, C_{2}>0\left|C_{1}\right| f(x)\left|\leq g(x) \leq C_{2}\right| f(x) \mid$, for all the values of $x$ for which this makes sense. For convenience, when dealing with orders of symbols, we will often use the obvious notations $e=(1,1), e_{1}=(1,0)$ and $e_{2}=(0,1)$. In general, $\psi$ dos will be denoted by capital letters and their symbols or amplitudes by the corresponding small letter (i.e., $P=\mathrm{Op}(p), q=$ $\operatorname{Sym}(Q)$, etc.). $A=A_{\varphi, a}$ and $B=B_{\varphi, b}$ will be respectively, unless otherwise stated, FIOs of Type I and II with phase function $\varphi$ and amplitudes $a$ and $b$.

Definition 2.1 For $m=\left(m_{1}, m_{2}, m_{3}\right) \in \mathbb{R}^{3}$, we denote by $\mathbf{S G}^{m}=\mathbf{S G}^{m}\left(\mathbb{R}^{n}\right)$ the space of all amplitudes functions $a \in C^{\infty}\left(\mathbb{R}^{n} \times \mathbb{R}^{n} \times \mathbb{R}^{n}\right)$ which satisfy the condition

$$
\begin{aligned}
& \forall \alpha, \beta, \gamma \in \mathbb{N}^{n} \exists C_{\alpha \beta \gamma}>0: \forall x, y, \xi \in \mathbb{R}^{n} \\
& \quad\left|\partial_{\xi}^{\alpha} \partial_{\beta}^{x} \partial_{\gamma}^{y} a(x, y, \xi)\right| \leq C_{\alpha \beta \gamma}\langle\xi\rangle^{m_{1}-|\alpha|}\langle x\rangle^{m_{2}-|\beta|}\langle y\rangle^{m_{3}-|\gamma|} .
\end{aligned}
$$

$\mathbf{S G}^{m}\left(\mathbb{R}^{n}\right)$ is given the usual Fréchet topology based upon the seminorms implicit in (2.3). When a is vector or matrix valued, $a \in \mathbf{S G}^{m}$ means that the estimates (2.3) are fulfilled component by component.

Definition 2.2 For $m=\left(m_{1}, m_{2}\right) \in \mathbb{R}^{2}$, we denote by $\mathbf{S G}_{l}^{m}=\mathbf{S G}_{l}^{m}\left(\mathbb{R}^{n}\right)$ the double-order symbol space of functions $a \in \mathbf{S G}^{\left(m_{1}, m_{2}, 0\right)}$ which are independent of $y$.

The following lemma is a result about compositions of elements of the $\mathbf{S G}$ classes of symbols and amplitudes. It is a basic tool in the proof of the theorems of composition among pseudodifferential and Fourier Integral operators.

Lemma 2.3 Let $f \in \mathbf{S G}^{m}$ and $g$ vector valued in $\mathbb{R}^{n}$ such that $g \in \mathbf{S G}^{e_{1}}$ and $\langle g(x, y, \xi)\rangle \sim\langle\xi\rangle$. Then $f(x, y, g(x, y, \xi)) \in \mathbf{S G}^{m}$.

Definition 2.4 With each amplitude $p \in \mathbf{S G}^{m}$ associate a linear operator $P=$ $\mathrm{Op}(p): \mathcal{S}\left(\mathbb{R}^{n}\right) \rightarrow \mathcal{S}\left(\mathbb{R}^{n}\right)$ defined as

$$
P u(x)=\operatorname{Op}(p) u(x)=\iint e^{i\langle x-y \mid \xi\rangle} p(x, y, \xi) u(y) d y d \xi .
$$


Here, as usual, $\vec{d} \xi=(2 \pi)^{-n} d \xi$, while $\langle z \mid \zeta\rangle$ denotes the scalar product of the two vectors $z, \zeta \in \mathbb{R}^{n}$. If the conditions $r \in \mathbb{R}^{2}$ and $q \in \mathbf{S G}_{l}^{r}$ hold ${ }^{3}$, (2.4) reduces to

$$
O p(p) u(x)=\int e^{i\langle x \mid \xi\rangle} q(x, \xi) \hat{u}(\xi) d \xi,
$$

where $\hat{u}(\xi)=\mathcal{F}_{x \rightarrow \xi}(u)(\xi)$ is the Fourier transform of $u$. Let us denote by $\mathbf{L G}^{r}$ the space of all the operators defined as in (2.5). An element $P \in \mathbf{L G}^{r}$ is called a $\mathbf{S G} \psi d o$, of order less or equal to $r$.

The $\psi \operatorname{dos}$ in $\mathbf{L G}^{r}$ form an algebra ${ }^{4}$ of linear continuous operators from $\mathcal{S}\left(\mathbb{R}^{n}\right)$ to $\mathcal{S}\left(\mathbb{R}^{n}\right)$, extendable as linear continuous operators from $\mathcal{S}^{\prime}\left(\mathbb{R}^{n}\right)$ to $\mathcal{S}^{\prime}\left(\mathbb{R}^{n}\right)$. The usual property about the order of the composed operators holds normally, simply understanding the sum of orders as a sum of vectors in $\mathbb{R}^{2}$. The residual elements of this $\psi$ dos algebra (smoothing operators) are integral operators with kernel in $\mathcal{S}\left(\mathbb{R}^{n} \times \mathbb{R}^{n}\right)$, whose set is denoted by $\mathcal{K}$. Moreover, it is also possible to prove that each operator defined by (2.4) with $p \in \mathbf{S G}^{m}, m=\left(m_{1}, m_{2}, m_{3}\right) \in$ $\mathbb{R}^{3}$, can be represented, modulo $\mathcal{K}$, as an operator of the form $(2.5)$ with $q \in$ $\mathbf{S G}_{l}^{r}, r=\left(m_{1}, m_{2}+m_{3}\right)$. Finally, it is easy to see that $\mathcal{K}$ coincides with the space of operators $\mathbf{L} \mathbf{G}^{-\infty}$, generated, equivalently, by amplitudes in $\mathbf{S G}^{-\infty}=$ $\bigcap_{m \in \mathbb{R}^{3}} \mathbf{S G}^{m}=\mathcal{S}\left(\mathbb{R}^{3 n}\right)$ or symbols in $\mathbf{S G}_{l}^{-\infty}=\bigcap_{m \in \mathbb{R}^{2}} \mathbf{S G}_{l}^{m}=\mathcal{S}\left(\mathbb{R}^{2 n}\right)$. For $P \in \mathbf{L G}^{m}$ we denote by $p=\operatorname{Sym}(P) \in \mathbf{S G}_{l}^{m}$ the symbol of $P$, that is $P=$ Op $(p)$. Moreover, we denote by $\operatorname{Sym}_{\mathrm{p}}(P)$ a principal symbol of $P$, that is a $p^{\prime} \in \mathbf{S G}_{l}^{m}$ such that $p-p^{\prime} \in \mathbf{S G}_{l}^{m-e}$.

In the present situation, the notion of asymptotic expansion is the following: for $a \in \mathbf{S G}_{l}^{m}, a_{j} \in \mathbf{S G}_{l}^{m-j e}, a \sim \sum_{j \in \mathbb{N}} a_{j} \Leftrightarrow \forall N \in \mathbb{N} a-\sum_{j=0}^{N} a_{j} \in$ $\mathbf{S G}_{l}^{m-(N+1) e}$. It is a fact that with any sequence of symbols with orders decreasing to $(-\infty,-\infty)$ it is possible to associate an asymptotic sum, which is unique modulo $\mathcal{S}\left(\mathbb{R}^{n} \times \mathbb{R}^{n}\right)$. We now recall the notion of ellipticity in this context. The elliptic $\mathbf{S G}$ operators have the usual properties of "invertibility modulo smoothing operators".

Definition 2.5 A symbol $p \in \mathbf{S G}_{l}^{m}$ and the corresponding operator $P=\mathrm{Op}(p)$ are called md-elliptic (or elliptic, for short) if exists $R>0$ such that

$$
|x|+|\xi|>R \Rightarrow p(x, \xi) \neq 0
$$

and

$$
|x|+|\xi|>R \Rightarrow \exists C>0:|p(x, \xi)|^{-1} \leq C\langle\xi\rangle^{-m_{1}}\langle x\rangle^{-m_{2}} .
$$

Let us denote by $\mathbf{E S G}_{l}^{m}=\mathbf{E} \mathbf{S G}_{l}^{m}\left(\mathbb{R}^{n}\right)$ the subset of $\mathbf{S G}_{l}^{m}$ of all md-elliptic symbols of order $m$ and by $\mathbf{E} \mathbf{L G}^{m}=\mathrm{Op}\left(\mathbf{E S G}_{l}^{m}\right)$ the corresponding subset of md-elliptic operators. Analogously, an amplitude $p \in \mathbf{S G}^{m}$ and the corresponding operator $P=\mathrm{Op}(p)$ are called md-elliptic if

$$
\exists \tilde{p} \in \mathbf{E S G}_{l}^{m^{\prime}}, m^{\prime}=\left(m_{1}, m_{2}+m_{3}\right) \mid \mathrm{Op}(p)-\mathrm{Op}(\tilde{p}) \in \mathcal{K} .
$$

\footnotetext{
${ }^{3}$ That is, the amplitude is, indeed, a symbol.

${ }^{4}$ Details about the $\mathbf{S G}$ calculus can be found in [2], [11] and the references quoted therein.
} 
Theorem 2.6 Each $P \in \mathbf{E L G}^{m}$ admits a $\mathcal{K}$-parametrix (or simply parametrix), that is, a $\psi d o Q \in \mathbf{L G}^{-m}$ such that

$$
P Q-I, Q P-I \in \mathcal{K}
$$

where I denotes the identity operator.

In the following definition, we recall the notion of the weighted Sobolev spaces "adapted" to the $\mathbf{S G}$ calculus.

Definition 2.7 For $s=\left(s_{1}, s_{2}\right) \in \mathbb{R}^{2}$, let $\pi_{s}$ denote the product $\pi_{s}(x, \xi)=$ $\langle\xi\rangle^{s_{1}}\langle x\rangle^{s_{2}}$ and $\Pi_{s}=\mathrm{Op}\left(\pi_{s}\right)$ the corresponding operator. The weighted Sobolev spaces $H^{s}\left(\mathbb{R}^{n}\right)=H^{s}, s=\left(s_{1}, s_{2}\right) \in \mathbb{R}^{2}$, are defined by:

$$
H^{s}=\left\{u \in \mathcal{S}^{\prime}\left(\mathbb{R}^{n}\right) \mid \Pi_{s} u \in L^{2}\left(\mathbb{R}^{n}\right)\right\},
$$

with the natural Hilbert norm $\|u\|_{s}=\left\|\Pi_{s} u\right\|_{L^{2}}=\left\|\Pi_{s} u\right\|_{0}$.

The SG $\psi$ dos act continuously on the spaces $H^{s}$, that is, $P \in \mathbf{L G}^{r}$ implies that $P$ is linear and continuous from $H^{s}$ to $H^{s-r}$ for all $r, s \in \mathbb{R}^{2}$.

We recall now the definition of SG FIO and state the main composition result among general SG $\psi$ dos and FIOs, which corresponds to Theorem 1.1 in the non-classical situation. The proof, together with a complete analysis of the properties of SG FIOs, can be found in [4] or [6].

Definition 2.8 We will call a phase function or simply phase any real valued $\varphi \in \mathbf{S G}_{l}^{e}$ satisfying

$$
\text { and } \begin{aligned}
c\langle x\rangle & \leq\left\langle\nabla_{\xi} \varphi\right\rangle \leq C\langle x\rangle \\
c\langle\xi\rangle & \leq\left\langle d_{x} \varphi\right\rangle \leq C\langle\xi\rangle
\end{aligned}
$$

for suitable constants $C, c>0$ and denote by $\mathcal{P}$ the set of all such phases. Moreover, we define the set $\mathcal{P}^{\epsilon}, \epsilon>0$ of all regular phases as follows:

$$
\mathcal{P}^{\epsilon}=\left\{\varphi \in \mathcal{P}|\forall x, \xi:| \operatorname{det}\left(\partial_{i}^{x} \partial_{\xi}^{j} \varphi\right) \mid \geq \epsilon\right\} .
$$

Definition 2.9 For any choice of $\varphi \in \mathcal{P}, a, b \in \mathbf{S G}_{l}^{m}$ and $u \in \mathcal{S}$, we define $a$ FIO of Type I as

$$
A u(x)=A_{\varphi, a} u(x)=\int e^{i \varphi(x, \xi)} a(x, \xi) \hat{u}(\xi) d \xi,
$$

and a FIO of Type II as

$$
\widehat{B u}(\xi)=\widehat{B_{\varphi, b}} u(\xi)=\int e^{-i \varphi(x, \xi)} \overline{b(x, \xi)} u(x) d x .
$$

Remark 2.10 The estimates (2.11) can be valid only outside a set of the type $|x|+|\xi| \geq R>0$ and all the results listed here remain true. 
Theorem 2.11 Let be given a FIO $A=A_{\varphi, a}$ of Type I such that $\varphi \in \mathcal{P}$ and $a \in \mathbf{S G}_{l}^{r}$ and $a \psi d o P=\mathrm{Op}(p)$ with $p \in \mathbf{S G}_{l}^{s}$. Then, the composed operator $H=P A$ is, modulo smoothing operators, a FIO of Type I. In fact, $H=H_{\varphi, h}$ where $\varphi$ is the same phase function and the amplitude $h \in \mathbf{S G}_{l}^{r+s}$ admits the following asymptotic expansion:

$$
h(x, \xi) \sim \sum_{\alpha \in \mathbb{N}^{n}} \frac{1}{\alpha !}\left(\partial_{\xi}^{\alpha} p\right)\left(x, d_{x} \varphi(x, \xi)\right) D_{\alpha}^{y}\left[e^{i \psi(x, y, \xi)} a(y, \xi)\right]_{y=x} .
$$

Here

$$
\psi(x, y, \xi)=\varphi(y, \xi)-\varphi(x, \xi)-\left\langle y-x \mid d_{x} \varphi(x, \xi)\right\rangle,
$$

and, as usual, $D_{\alpha}^{y}=(-i)^{|\alpha|} \partial_{\alpha}^{y}$. We will write $h=p \circ_{\varphi}$ a as a short form of (2.15).

Similar results hold for the composition $A P$ and for the composition among SG $\psi$ dos operators and SG FIOs of Type II (see [4] or [6]).

We now introduce the subclass of the Classical SG symbols. They can be defined as follows ${ }^{5}$.

Definition 2.12 Let $S^{m}, m \in \mathbb{R}$, denote the space of global classical symbols in one variable. This means that $a \in S^{m}$ if $a=a(\xi)$ is smooth on $\mathbb{R}^{n}$, satisfies estimates like (1.1) in the only variable $\xi$ and there exist functions $a_{(m-j)} \in$ $C^{\infty}\left(\mathbb{R}^{n} \backslash\{0\}\right), j \in \mathbb{N}$, homogeneous of degree $m-j$, such that, for some excision function $^{6} \omega$, we have

$$
a(\xi) \sim \sum_{j=0}^{\infty} \omega(\xi) a_{j}(\xi)
$$

Then, for $m=\left(m_{1}, m_{2}\right) \in \mathbb{R}^{2}, \mathbf{S G}_{l, c l}^{m}=S_{\xi}^{m_{1}} \hat{\otimes}_{\pi} S_{x}^{m_{2}}$.

An equivalent definition by means of asymptotic expansions in terms of subclasses of homogeneous SG symbols can be found in [8], which we refer to for most of the notations concerning the classical SG symbols ${ }^{7}$. It easily turns out that classical symbols are closed under sums and products. Moreover, with any $a \in \mathbf{S G}_{l \mathrm{cl}}^{m}$ we can canonically associate its principal symbol ${ }^{8}$ $\operatorname{Sym}_{\mathrm{p}}^{\mathrm{cl}}(a)=\left\{\sigma_{\psi}^{m_{1}}(a) ; \sigma_{e}^{m_{2}}(a), \sigma_{\psi, \mathrm{e}}^{m}(a)\right\} \cdot \sigma_{\psi}^{m_{1}}(a)$ is called the homogeneous principal interior symbol and the pair $\left\{\sigma_{e}^{m_{2}}(a), \sigma_{\psi, \mathrm{e}}^{m}(a)\right\}$ the homogeneous principal exit symbol of $a$.

\footnotetext{
${ }^{5}$ See $[15]$.

${ }^{6}$ That is, $\omega(\xi) \in C^{\infty}\left(\mathbb{R}^{n},[0,1]\right)$ is zero in a neighbourhood of the origin and is 1 for large values of $|\xi|$.The notion of asymptotic equivalence here is analogous to the $\mathbf{S G}$ one, but in a single variable.

${ }^{7}$ Our $\mathbf{S G}_{l, c l}^{\left(m_{1}, m_{2}\right)}$ is denoted by $S_{\mathrm{cl}(x, \xi)}^{m_{1}, m_{2}}$ in the cited book. We also write $\sigma_{\psi, \mathrm{e}}^{m}$ with $m \in \mathbb{R}^{2}$ rather than $\sigma_{\psi, e}^{m_{1}, m_{2}}$.

${ }^{8}$ See again [8] (compare also with [9]). Any reference to the total or exit principal symbol must be understood component by component. So, saying that $\operatorname{Sym}_{\mathrm{p}}(a)$ is real-valued means that all the three components share this property, etc.
} 
To our aim, the following two results, included in [8], are central. The first says that, just like for the general SG symbols, classical symbols are identified by asymptotic expansion. Through the second one, we obtain the description of the topology of the classical symbols spaces.

Theorem 2.13 Let $a_{k} \in \mathbf{S G}_{l, c l}^{m-k e}, k \in \mathbb{N}$ be an arbitrary sequence of classical symbols and $a \sim \sum_{k=0}^{\infty} a_{k}$ the asymptotic sum in the sense of the general $\mathbf{S G}$ symbols, as recalled in section 2. Then, $a \in \mathbf{S G}_{l, c l}^{m}$.

Theorem 2.14 Let $B^{n}=\left\{x \in \mathbb{R}^{n}:|x| \leq 1\right\}$ and let $\chi$ be a diffeomorphism from the interior of $B^{n}$ to $\mathbb{R}^{n}$ such that $|x|>2 / 3 \Rightarrow \chi(x)=\frac{x}{|x|(1-|x|)}$. Choosing a $C^{\infty}$ function $[x]$ on $\mathbb{R}^{n}$ such that $1-[x] \neq 0$ for all $x$ in the interior of $B^{n}$ and $|x|>2 / 3 \Rightarrow[x]=|x|$, for any $a \in \mathbf{S G}_{l, c l}^{m}$ denote by $\left(D^{m} a\right)(y, \eta)$ the function

$$
b(y, \eta)=(1-[\eta])^{m_{1}}(1-[y])^{m_{2}} a(\chi(y), \chi(\eta)) .
$$

Then, $D^{m}$ extends to a homeomorphism from $\mathbf{S G}_{l, \mathrm{cl}}^{m}$ to $C^{\infty}\left(B^{n} \times B^{n}\right)$.

Remark 2.15 General FIO calculi, including the SG FIOs calculus, already existed (see, e.g., [10] and the references quoted therein). We preferred, in view of our applications, a more transparent approach, completely in the $\mathbf{S G}$ environment. At our knowledge, however, the present classical SG FIOs have not yet been studied.

\section{Proof of the composition theorem}

We will prove the part of the statement of Theorem 1.1 concerning the composition $P A$. The remaining results can be proved as in [4]. Owing to Theorem 2.11 , we only need to show that $h \in \mathbf{S G}_{l, c l}^{r+s}$. We will obtain this result through different steps, dealing with compositions of classical symbols. The following Lemma is immediate, by the natural inclusions among the classical symbols spaces.

Lemma 3.1 Let $\varphi \in \mathbf{S G}_{l, c l}^{e}$. Then, for $\psi(x, y, \xi)$ defined in (2.16), we have

$$
\left.D_{\beta}^{y} e^{i \psi(x, y, \xi)}\right|_{y=x} \in \mathbf{S G}_{l, c l}^{(E(|\beta| / 2), E(-|\beta| / 2))} .
$$

Definition 3.2 We denote by $\mathcal{P}_{c l}$ the subset of classical phase functions. It is defined as the set of all $\varphi \in \mathcal{P}$ such that $\varphi \in \mathbf{S G}_{l, c l}^{e}$. Moreover, by $\mathcal{P}_{c l}^{\epsilon}$ we denote the subset of regular classical phase functions. Using the notations above, we simply set

$$
\mathcal{P}_{c l}^{\epsilon}=\left\{\varphi \in \mathcal{P}_{c l} \mid \varphi \in \mathcal{P}^{\epsilon}\right\}
$$

A classical SG FIO (of Type I or Type II) is any SG FIO defined as in (2.13) or (2.14) such that $\varphi \in \mathcal{P}_{c l}$ and $a, b \in \mathbf{S G}_{l, c l}^{m}$. A classical $\mathbf{S G}$ FIO will be called elliptic if its phase function is classical regular and its amplitude is classical elliptic. 
Proposition 3.3 If $p \in \mathbf{S G}_{l, \mathrm{cl}}^{m}$ and $\omega \in \mathbf{S G}_{l, \mathrm{cl}}^{e_{1}}$ is a vector valued classical symbol such that $\langle\omega(x, \xi)\rangle \sim\langle\xi\rangle$, we have $q(x, \xi)=p(x, \omega(x, \xi)) \in \mathbf{S G}_{l, \mathrm{cl}}^{m}$.

Proof. Consider the topological isomorphism defined in Theorem 2.14. $q \in$ $\mathbf{S G}_{l, c l}^{m}$ is equivalent to $\tilde{q}=D^{m} q \in C^{\infty}\left(B^{n} \times B^{n}\right)$. But we have, by definition of $D^{m} q$,

$$
\begin{aligned}
\tilde{q}(y, \eta)= & (1-[\eta])^{m_{1}}(1-[y])^{m_{2}} p(\chi(y), \omega(\chi(y), \chi(\eta))) \\
= & \left(\frac{1-[\eta]}{1-\left[\chi^{-1}(\omega(\chi(y), \chi(\eta)))\right]}\right)^{m_{1}} . \\
& \left(1-\left[\chi^{-1}(\omega(\chi(y), \chi(\eta)))\right]\right)^{m_{1}}(1-[y])^{m_{2}} . \\
& p\left(\chi(y), \chi\left(\chi^{-1}(\omega(\chi(y), \chi(\eta)))\right)\right) \\
= & \left(\frac{1-[\eta]}{1-\left[\chi^{-1}\left(\frac{\tilde{\omega}(y, \eta)}{1-[\eta]}\right)\right]}\right)^{m_{1}} \tilde{p}\left(y, \chi^{-1}\left(\frac{\tilde{\omega}(y, \eta)}{1-[\eta]}\right)\right),
\end{aligned}
$$

where we set $\tilde{p}=D^{m} p$ and $\tilde{\omega}=D^{e_{1}} \omega$. So, we just have to prove that the two factors are smooth functions up to the boundary on $B^{n} \times B^{n}$. Let us consider, first of all, the expression $f(y, \eta)=\chi^{-1}\left(\frac{\tilde{\omega}(y, \eta)}{1-[\eta]}\right)$. Observe that this function is smooth in the product of the interiors of the two balls $B^{n}$. This is due to the choice of $[\eta]$ and $\chi$ and to the fact that $\omega \in \mathbf{S G}_{l, c l}^{e_{1}} \Rightarrow \tilde{\omega} \in C^{\infty}\left(B^{n} \times B^{n}\right)$. So we need only to consider $f(y, \eta)$ for $|y| \rightarrow 1^{-}$or $|\eta| \rightarrow 1^{-}$. It is clear that $f$ is smooth up to $|y|=1$ for all $\eta$ in the interior of $B^{n}$. Now, observe the following: $\langle\omega(x, \xi)\rangle \sim\langle\xi\rangle \Rightarrow|\tilde{\omega}(y, \eta)| \geq c>0$ for $|\eta|$ close enough to 1 . Indeed, assuming $|\eta|>2 / 3$,

$$
\begin{aligned}
& \langle\omega(x, \xi)\rangle \sim\langle\xi\rangle \Leftrightarrow\left\langle\frac{\tilde{\omega}(y, \eta)}{1-[\eta]}\right\rangle \sim\langle\chi(\eta)\rangle=\left\langle\frac{\eta}{|\eta|(1-|\eta|)}\right\rangle \Leftrightarrow \\
\Leftrightarrow & 1+\frac{|\tilde{\omega}(y, \eta)|^{2}}{(1-|\eta|)^{2}} \geq 2 c^{2}+\frac{2 c^{2}}{(1-|\eta|)^{2}} \Leftrightarrow|\tilde{\omega}(y, \eta)|^{2} \geq 2 c^{2}+\left(2 c^{2}-1\right)(1-|\eta|)^{2} \Rightarrow \\
\Rightarrow & |\tilde{\omega}(y, \eta)|^{2} \geq c^{2} \Rightarrow|\tilde{\omega}(y, \eta)| \geq c
\end{aligned}
$$

for $|\eta| \rightarrow 1^{-}$. So, we have $|\eta| \rightarrow 1^{-} \Rightarrow\left|\frac{\tilde{\omega}(y, \eta)}{1-[\eta]}\right| \rightarrow+\infty$. Then, for $|\eta| \rightarrow 1^{-}$we can use the explicit expression of $\chi^{-1}$, obtaining

$$
f(y, \eta)=\frac{\frac{|\tilde{\omega}(y, \eta)|}{1-[\eta]}-1}{\frac{|\tilde{\omega}(y, \eta)|^{2}}{(1-[\eta])^{2}}} \frac{\tilde{\omega}(y, \eta)}{1-[\eta]}=\frac{|\tilde{\omega}(y, \eta)|-(1-|\eta|)}{|\tilde{\omega}(y, \eta)|^{2}} \tilde{\omega}(y, \eta) \in C^{\infty}\left(B^{n} \times B^{n}\right),
$$

by the above observations. Being a composition of two functions in $C^{\infty}\left(B^{n} \times\right.$ $\left.B^{n}\right)$, the second factor in (3.3) is itself in $C^{\infty}\left(B^{n} \times B^{n}\right)$. Let us now consider 
the first factor in (3.3). Again, by the analysis carried out above and by the choices of $[\eta]$ and $\chi$, this is a smooth function in the product of the interiors, and, indeed, up to $|y|=1$. For $|\eta| \geq r>0$ and $r \in(0,1)$ close enough to 1 , reasoning as above, we easily get

$$
\left(\frac{1-[\eta]}{1-\left[\chi^{-1}\left(\frac{\tilde{\omega}(y, \eta)}{1-[\eta]}\right)\right]}\right)^{m_{1}}=|\tilde{\omega}(y, \eta)|^{m_{1}}
$$

Since we have proved that in the region we are considering $|\tilde{\omega}(y, \eta)| \geq c>0$, the first factor of (3.3), too, is in $C^{\infty}\left(B^{n} \times B^{n}\right)$. This completes the proof.

The following result is the final step for the proof of Theorem 1.1

Theorem 3.4 For all $p \in \mathbf{S G}_{l, c l}^{m}, a \in \mathbf{S G}_{l, c l}^{t}, \varphi \in \mathcal{P}_{c l}$, we have $h=p \circ_{\varphi} a \in$ $\mathbf{S G}_{l, c l}^{m+t}$.

Proof. Our assumptions imply that $\omega=d_{x} \varphi \in \mathbf{S G}_{l, c l}^{e_{1}}$ satisfies all the requirements of Proposition 3.3 above, so that we have

$$
\forall \alpha \in \mathbb{N}^{n}\left(\partial_{\xi}^{\alpha} p\right)\left(x,\left(d_{x} \varphi\right)(x, \xi)\right) \in \mathbf{S G}_{l, c l}^{m-|\alpha| e_{1}} .
$$

Then, the desired result follows by the multiplication properties of classical symbols, by (2.15), (3.1) and (3.5) and by Theorem 2.13 .

\section{The Cauchy Problem in the SG classical en- vironment}

In this section we apply the theory of classical SG FIOs to the solution of the Cauchy problem for a classical $\mathbf{S G} \psi$ do of order $e$, similarly to what was done in [5]. The differences in the present treatment amount to the proof that the involved objects are classical in the above sense.

Consider a symbol $\lambda \in C^{\infty}\left(J ; \mathbf{S G}_{l, c l}^{e}\right), J=[-T, T], T>0$, which we take scalar-valued, for simplicity. Choosing $u_{0} \in H^{s}, s \in \mathbb{R}^{2}$, we already know that, if $\Lambda(t)=\mathrm{Op}(\lambda(t ; . .)$.$) is hyperbolic (i.e., if there exists a real-valued \lambda_{e}$ such that $\left.\lambda_{0}=\lambda-\lambda_{e} \in C^{\infty}\left(J ; \mathbf{S G}_{l}^{0}\right)\right)$, the solution of

$$
\left\{\begin{array}{l}
\left(D_{t}-\Lambda(t)\right) u(t)=0 \quad t \in J \\
u(0)=u_{0}
\end{array}\right.
$$

exists and is unique. We also know that $u(t)=A_{\varphi(t), a(t)} u_{0}$, with $A(t)=$ $A_{\varphi(t), a(t)}$ SG FIO of Type I, depending smoothly on $t \in J^{\prime}=\left[-T^{\prime}, T^{\prime}\right] \subseteq J$, $T^{\prime}>0$. Note that $\operatorname{Sym}_{\mathrm{p}}^{\mathrm{cl}}(\lambda)$ real-valued implies $\Lambda$ hyperbolic: indeed, we can take $^{9}$

$$
\lambda_{e}(t ; x, \xi)=\omega(\xi) \sigma_{\psi}^{1}(\lambda)(t ; x, \xi)+\omega(x)\left(\sigma_{e}^{1}(\lambda)(t ; x, \xi)-\omega(\xi) \sigma_{\psi, e}^{(1,1)}(\lambda)(t ; x, \xi)\right),
$$

\footnotetext{
${ }^{9}$ See $[14]$.
} 
where $\omega \in C^{\infty}\left(\mathbb{R}^{n} ;[0,1]\right)$ is an excision function ${ }^{10}$.

We divide the proof of Theorem 1.2 in various steps, analysing separately the solution of the eikonal and of the transport equations, as in [5]. We deal first with the Hamiltonian system (4.3) associated with the principal symbol of $\lambda$, since, as it is well known, the solution of the eikonal equation

$$
\left\{\begin{array}{l}
\frac{\partial \varphi}{\partial t}(t ; x, \xi)=\lambda_{e}\left(x,\left(d_{x} \varphi\right)(t ; x, \xi)\right) \\
\varphi(0 ; x, \xi)=\langle x \mid \xi\rangle
\end{array}\right.
$$

can be given in terms of the solution of such a system.

Proposition 4.1 Let $\lambda_{e} \in C^{\infty}\left(J ; \mathbf{S G}_{l, \mathrm{cl}}^{e}\right)$. The solution $(q(t ; x, \xi), p(t ; x, \xi))$ of the Hamiltonian system

$$
\left\{\begin{array}{l}
\dot{q}(t ; x, \xi)=\left(\nabla_{\xi} \lambda_{e}\right)(t ; q(t ; x, \xi), p(t ; x, \xi)) \\
\dot{p}(t ; x, \xi)=\left(-d_{x} \lambda_{e}\right)(t ; q(t ; x, \xi), p(t ; x, \xi)) \\
q(0 ; x, \xi)=x \\
p(0 ; x, \xi)=\xi
\end{array}\right.
$$

satisfies $q \in C^{\infty}\left(J^{\prime} ; \mathbf{S G}_{l, c l}^{e_{2}}\right)$ and $p \in C^{\infty}\left(J^{\prime} ; \mathbf{S G}_{l, c l}^{e_{1}}\right)$ with $J^{\prime}=\left[-T^{\prime}, T^{\prime}\right] \subseteq J$, $T^{\prime}>0$.

Proof. The solution of (4.3) can be found by means of the iterative scheme

$$
\left\{\begin{array}{l}
q_{k+1}(t ; x, \xi)=x+\int_{0}^{t}\left(\nabla_{\xi} \lambda_{e}\right)\left(s ; q_{k}(s ; x, \xi), p_{k}(s ; x, \xi)\right) d s \\
p_{k+1}(t ; x, \xi)=\xi-\int_{0}^{t}\left(d_{x} \lambda_{e}\right)\left(s ; q_{k}(s ; x, \xi), p_{k}(s ; x, \xi)\right) d s
\end{array}\right.
$$

We will prove the following:

1. $\forall k \in \mathbb{N} q_{k}(t ;.) \in C^{\infty}\left(J^{\prime}, \mathbf{S G}_{l, c l}^{e_{2}}\right), p_{k}(t ;.) \in C^{\infty}\left(J^{\prime}, \mathbf{S G}_{l, c l}^{e_{1}}\right)$, for some $J^{\prime}=$ $\left[-T^{\prime}, T^{\prime}\right] \subseteq J$

2. The sequences $\left\{q_{k}(t ;).\right\}$ and $\left\{p_{k}(t ;).\right\}$ are convergent in the topology of the respective classical symbol classes, i.e., the solutions $q(t ;$.$) and p(t ;$. are classical symbols with the corresponding order.

To this aim we will use a technique similar to that used in the proof of Proposition 3.3 , by means of the topological isomorphism induced by (2.17). We already know (see [2] and [5]) that the solution is globally defined on $J$; moreover, $\langle q(t ; x, \xi)\rangle \sim\langle x\rangle$ and $\langle p(t ; x, \xi)\rangle \sim\langle\xi\rangle$, uniformly for $t \in J$. This is also true for any $q_{k}(t ;$.$) and p_{k}(t ;$.$) , possibly restricting J$ to $J^{\prime}=\left[-T^{\prime}, T^{\prime}\right]$, as we will now prove by induction ${ }^{11}$. In fact, recall that, with suitable $C_{10}, C_{01}>0$,

$$
\left|d_{x} \lambda_{e}(t ; x, \xi)\right| \leq C_{10}\langle\xi\rangle,\left|\nabla_{\xi} \lambda_{e}(t ; x, \xi)\right| \leq C_{01}\langle x\rangle,
$$

\footnotetext{
${ }^{10}$ That is, $\omega(x)$ is zero in a neighborhood of the origin and is 1 for large values of $|x|$.

${ }^{11}$ This part of the proof is essentially the same contained in [1].
} 
and that

$$
\exists C>0 \exists c_{1} \in(0,1) \exists c_{2}>1:|y-x| \leq C\langle x\rangle \Rightarrow c_{1}\langle x\rangle \leq\langle y\rangle \leq c_{2}\langle x\rangle .
$$

Then, setting $T^{\prime}=\min \left\{T, \frac{C}{c_{2} C_{10}}, \frac{C}{c_{2} C_{01}}\right\}$, we immediately see that $q_{1} \in$ $C^{\infty}\left(J^{\prime} ; \mathbf{S G}_{l, c l}^{e_{2}}\right), p_{1} \in C^{\infty}\left(J^{\prime} ; \mathbf{S G}_{l, c l}^{e_{1}}\right)$ and,$\forall t \in J^{\prime}$,

$$
\begin{aligned}
& \left|q_{1}(t ; x, \xi)-x\right| \leq C_{01}|t|\langle x\rangle \leq C\langle x\rangle, \\
& \left|p_{1}(t ; x, \xi)-\xi\right| \leq C_{10}|t|\langle\xi\rangle \leq C\langle\xi\rangle,
\end{aligned}
$$

implying $\left\langle q_{1}(t ; x, \xi)\right\rangle \sim\langle x\rangle,\left\langle p_{1}(t ; x, \xi)\right\rangle \sim\langle\xi\rangle$ (uniformly for $t \in J^{\prime}$ ). It remains to prove that if such a uniform estimate holds for the $(k-1)$-th iteration, then the same holds for the $k$-th one. Actually we get

$$
\begin{aligned}
& \left|q_{k}(t ; x, \xi)-x\right| \leq C_{01}\left|\int_{0}^{t}\left\langle q_{k-1}(s ; x, \xi)\right\rangle d s\right| \leq C_{01} T^{\prime} c_{2}\langle x\rangle \leq C\langle x\rangle, \\
& \left|p_{k}(t ; x, \xi)-\xi\right| \leq C_{10}\left|\int_{0}^{t}\left\langle p_{k-1}(s ; x, \xi)\right\rangle d s\right| \leq C_{10} T^{\prime} c_{2}\langle\xi\rangle \leq C\langle\xi\rangle,
\end{aligned}
$$

so that we can conclude

$$
\begin{aligned}
& \left\langle q_{k}(t ; x, \xi)\right\rangle \sim\langle x\rangle, \\
& \left\langle p_{k}(t ; x, \xi)\right\rangle \sim\langle\xi\rangle,
\end{aligned}
$$

uniformly for all $k \in \mathbb{N}$ and $t \in J^{\prime}$. Since $\lambda_{e} \in C^{\infty}\left(J, \mathbf{S G}_{l, c l}^{e}\right)$, using Proposition 3.3 , we get point i) above. To prove point ii), we proceed as follows.

- We consider the sequences $\left\{\tilde{q}_{k}\right\}=\left\{D^{e_{2}} q_{k}\right\}$ and $\left\{\tilde{p}_{k}\right\}=\left\{D^{e_{1}} p_{k}\right\}$, which are made up of elements of $C^{\infty}\left(B^{n} \times B^{n}\right)$, owing to point i) above;

- We prove that each one of the sequences $\left\{\tilde{q}_{k}\right\}$ and $\left\{\tilde{p}_{k}\right\}$ has a limit point in $C^{\infty}\left(B^{n} \times B^{n}\right)$. Since $C^{\infty}\left(B^{n} \times B^{n}\right)$ is a nuclear Frechét space, in order to say that a subsequence of $\left\{\tilde{q}_{k}\right\}$ and $\left\{\tilde{p}_{k}\right\}$ converges in $C^{\infty}\left(B^{n} \times B^{n}\right)$ it is enough to check that each sequence is bounded. Let us denote by $\tilde{q}$ and $\tilde{p}$ the two limit points in $C^{\infty}\left(B^{n} \times B^{n}\right)$.

- Now, recall that $D^{e_{1}}$ and $D^{e_{2}}$ are topological isomorphisms and that the topologies of $\mathbf{S G}_{l, \mathrm{cl}}^{e_{1}}$ and $\mathbf{S G}_{l \mathrm{cl}}^{e_{2}}$ are stronger than those induced respectively by $\mathbf{S G}_{l}^{e_{1}}$ and $\mathbf{S G}_{l}^{e_{2}}$. So, since we already know that the sequences $\left\{q_{k}\right\}$ and $\left\{p_{k}\right\}$ converge in the respective symbol classes, we can conclude that for the solutions $q$ and $p$ we have $q=\left(D^{e_{2}}\right)^{-1} \tilde{q} \in \mathbf{S G}_{l, c l}^{e_{2}}$ and $p=\left(D^{e_{1}}\right)^{-1} \tilde{p} \in \mathbf{S G}_{l, c l}^{e_{1}}$, as claimed.

So we just have to check the boundedness of the seminorms of the elements of the sequences $\left\{\tilde{q}_{k}\right\}$ and $\left\{\tilde{p}_{k}\right\}$. Let us set

$$
\tilde{b}_{01}=D^{e_{2}}\left(\nabla_{\xi} \lambda_{e}\right), \tilde{b}_{10}=D^{e_{1}}\left(d_{x} \lambda_{e}\right),
$$




$$
\begin{aligned}
& \begin{array}{l}
g(y)=(1-[y]) \chi(y), y \in B^{n}, \\
\forall h \in C^{\infty}\left(B^{n} \times B^{n}\right) \forall N \in \mathbb{N} s_{N}(h)= \\
\begin{array}{c}
(y, \eta) \in B^{n} \times B^{n} \\
|\alpha+\beta| \leq N
\end{array}
\end{array}\left|\partial_{\eta}^{\alpha} \partial_{\beta}^{y} h(y, \eta)\right| \\
& c_{N}=s_{N}(g), \\
& a_{0}=\sup \left\{\sup _{t \in J^{\prime}} s_{0}\left(b_{01}(t ; ., . .)\right), \sup _{t \in J^{\prime}} s_{0}\left(b_{10}(t ; ., . .)\right)\right\} .
\end{aligned}
$$

We will give the detailed proof of the boundedness of the seminorms $s_{0}\left(\tilde{q}_{k}(t ; ., .).\right)$ and $s_{0}\left(\tilde{p}_{k}(t ; .,).\right)$, and some remarks about the proof of the result for higher order seminorms. Applying $D^{e_{2}}$ and $D^{e_{1}}$ to both sides of (4.4), respectively in the first and second row, and repeating some steps of the proof Proposition 3.3, we get,

$$
\left\{\begin{aligned}
& \tilde{q}_{k+1}(t ; y, \eta)=g(y)+\int_{0}^{t} \frac{1-[y]}{1-\left[\chi^{-1}\left(\frac{\tilde{q}_{k}(s ; y, \eta)}{1-[y]}\right)\right]} \cdot \\
& \tilde{p}_{k+1}(t ; y, \eta)=g(\eta)-\int_{0}^{t} \frac{\tilde{b}_{01}\left(s ; \chi^{-1}\left(\frac{\tilde{q}_{k}(s ; y, \eta)}{1-[y]}\right), \chi^{-1}\left(\frac{\tilde{p}_{k}(s ; y, \eta)}{1-[\eta]}\right)\right) d s}{1-\left[\chi^{-1}\left(\frac{\tilde{p}_{k}(s ; y, \eta)}{1-[\eta]}\right)\right]} \cdot \\
& . \tilde{b}_{10}\left(s ; \chi^{-1}\left(\frac{\tilde{q}_{k}(s ; y, \eta)}{1-[y]}\right), \chi^{-1}\left(\frac{\tilde{p}_{k}(s ; y, \eta)}{1-[\eta]}\right)\right) d s .
\end{aligned}\right.
$$

First of all, we prove that $s_{0}\left(q_{k}(t ; ., .).\right)$ and $s_{0}\left(p_{k}(t ; . .).\right)$ are uniformly bounded with respect to $k \in \mathbb{N}$ and $t \in J^{\prime}$. To this aim, we need to study the function

$$
g\left(y_{1}, y_{2}\right)=\frac{1-\left[y_{1}\right]}{1-\left[\chi^{-1}\left(\frac{y_{2}}{1-\left[y_{1}\right]}\right)\right]},
$$

which enters as first factor in each one of the two integrands of (4.9). From the proof of Proposition 3.3, we see that $|y| \rightarrow 1^{-} \Rightarrow\left|\tilde{q}_{k}(t ; y, \eta)\right| \geq c>0$ and $|\eta| \rightarrow 1^{-} \Rightarrow\left|\tilde{p}_{k}(t ; y, \eta)\right| \geq c>0$, uniformly with respect to $k \in \mathbb{N}$ and $t \in J^{\prime}$. So, when $y_{1}$ is close to 1 we need only to consider the values of $y_{2}$ such that $\left|y_{2}\right| \geq c>0$. By this observation, (3.4) and the choices of $\chi$ and $[y]$, we see that $g \in C^{\infty}(W)$, where ${ }^{12} W=\left\{\left(y_{1}, y_{2}\right) \in B^{n} \times \mathbb{R}^{n}|| y_{1} \mid \leq\right.$ $r\} \cup\left\{\left(y_{1}, y_{2}\right) \in B^{n} \times \mathbb{R}^{n}|| y_{1}|>r,| y_{2} \mid \geq c\right\}$. It is also easy to see that, on $W$, $\left|g\left(y_{1}, y_{2}\right)\right| \leq M+\left|y_{2}\right|$ with a suitable $M>0$. We proceed again by induction, obtaining, for any $t \in J^{\prime}$,

$$
\left\{\begin{array}{l}
s_{0}\left(\tilde{q}_{0}(t ; ., . .)\right)=c_{0} \\
s_{0}\left(\tilde{p}_{0}(t ; . . .)\right)=c_{0}
\end{array}\right.
$$

${ }^{12} r \in(0,1)$ is chosen in such a way that (3.4) holds. 


$$
\begin{aligned}
& \left\{\begin{array}{cl}
s_{0}\left(\tilde{q}_{1}(t ; ., . .)\right) & \leq\left(c_{0}+a_{0}\left(M+c_{0}\right)|t|\right) \leq\left(c_{0}\left(1+a_{0}|t|\right)+a_{0} M|t|\right) \\
s_{0}\left(\tilde{p}_{1}(t ; . . . .)\right) & \leq c_{0}\left(1+a_{0} T^{\prime}\right)+a_{0} M T^{\prime} \\
& \leq c_{0}\left(1+a_{0}|t|\right)+a_{0} M|t| \leq c_{0}\left(1+a_{0} T^{\prime}\right)+a_{0} M T^{\prime}
\end{array}\right. \\
& \left(s_{0}\left(\tilde{q}_{2}(t ; ., . .)\right) \leq\left(c_{0}+a_{0}\left|\int_{0}^{t}\left(M+c_{0}\left(1+a_{0}|s|\right)+a_{0} M|s|\right) d s\right|\right)\right. \\
& \leq c_{0}\left(1+a_{0}|t|+\frac{\left(a_{0}|t|\right)^{2}}{2}\right)+M\left(a_{0}|t|+\frac{\left(a_{0}|t|\right)^{2}}{2}\right) \\
& \left\{\begin{aligned}
\leq & c_{0}\left(1+a_{0} T^{\prime}+\frac{\left(a_{0} T^{\prime}\right)^{2}}{2}\right)+M\left(a_{0} T^{\prime}+\frac{\left(a_{0} T^{\prime}\right)^{2}}{2}\right) \\
s_{0}\left(\tilde{p}_{2}(t ; ., . .)\right) & \leq c_{0}\left(1+a_{0}|t|+\frac{\left(a_{0}|t|\right)^{2}}{2}\right)+M\left(a_{0}|t|+\frac{\left(a_{0}|t|\right)^{2}}{2}\right)
\end{aligned}\right. \\
& \leq c_{0}\left(1+a_{0} T^{\prime}+\frac{\left(a_{0} T^{\prime}\right)^{2}}{2}\right)+M\left(a_{0} T^{\prime}+\frac{\left(a_{0} T^{\prime}\right)^{2}}{2}\right) \\
& \left\{\begin{array}{l}
\cdots \\
\cdots
\end{array}\right.
\end{aligned}
$$

This gives

$$
\exists R=R\left(a_{0}, c_{0}, M, T^{\prime}\right)>0 \mid \forall k \in \mathbb{N} \forall t \in J^{\prime} s_{0}\left(\tilde{q}_{k}(t ; . . .)\right), s_{0}\left(\tilde{p}_{k}(t ; . . .)\right) \leq R
$$

as desired. Now, let us denote by $\tilde{h}_{01}$ and $\tilde{h}_{10}$ the whole integrands, respectively, of the first and second row of (4.9). Denoting by $B_{R}^{n}$ the ball in $\mathbb{R}^{n}$ of radius $R$ centered in the origin, by our previous considerations it turns out that $\tilde{h}_{01}, \tilde{h}_{10}$ are smooth functions of $(t ; y, \eta ; z, \zeta) \in J^{\prime} \times B^{n} \times B^{n} \times B_{R}^{n} \times B_{R}^{n}$, and the iteration scheme can be rewritten as

$$
\left\{\begin{array}{l}
\tilde{q}_{k+1}(t ; y, \eta)=g(y)+\int_{0}^{t} \tilde{h}_{01}\left(s ; y, \eta ; \tilde{q}_{k}(s ; y, \eta), \tilde{p}_{k}(s ; y, \eta)\right) d s \\
\tilde{p}_{k+1}(t ; y, \eta)=g(\eta)-\int_{0}^{t} \tilde{h}_{10}\left(s ; y, \eta ; \tilde{q}_{k}(s ; y, \eta), \tilde{p}_{k}(s ; y, \eta)\right) d s .
\end{array}\right.
$$

We conclude the proof observing that the uniform boundedness of $s_{N}\left(\tilde{q}_{k}(t ; ., .).\right)$ and $s_{N}\left(\tilde{p}_{k}(t ; ., .).\right), N \geq 1$, as well as of the higher order seminorms of the $t$ derivatives, can be proved in a similar way, keeping in mind the following facts:

- we can introduce the seminorms

$$
\tilde{a}_{N}=\sup \left\{\begin{array}{c}
\sup _{\substack{J^{\prime} \times B^{n} \times B^{n} \times B_{R}^{n} \times B_{R}^{n} \\
\left|\alpha_{1}+\beta_{1}+\alpha_{2}+\beta_{2}\right|=N}}\left|\partial_{\eta}^{\alpha_{1}} \partial_{\beta_{1}}^{y} \partial_{\zeta}^{\alpha_{2}} \partial_{\beta_{2}}^{z} h_{01}\right|, \\
\left.\sup _{\substack{J^{\prime} \times B^{n} \times B^{n} \times B_{R}^{n} \times B_{R}^{n} \\
\left|\alpha_{1}+\beta_{1}+\alpha_{2}+\beta_{2}\right|=N}}\left|\partial_{\eta}^{\alpha_{1}} \partial_{\beta_{1}}^{y} \partial_{\zeta}^{\alpha_{2}} \partial_{\beta_{2}}^{z} h_{10}\right|\right\} ;
\end{array}\right.
$$


- by the Fáa di Bruno formula, we see that the derivatives of $\tilde{q}_{k}(t ; y, \eta)$ and $\tilde{p}_{k}(t ; y, \eta)$ of total height $N \geq 1$ can be expressed by a formula similar to (4.12): the integrand is a polynomial in the derivatives of total height less or equal to $N-1$, linear in the derivatives of total height $N$; the polynomial coefficients are derivatives of $h_{01}$ and $h_{10}$, evaluated at $\left(t ; y, \eta ; \tilde{q}_{k}(t ; y, \eta), \tilde{p}_{k}(t ; y, \eta)\right) \in J^{\prime} \times B^{n} \times B^{n} \times B_{R}^{n} \times B_{R}^{n} ;$

- then, assuming that one has already proved the uniform boundedness of the seminorms $s_{j}\left(\tilde{q}_{k}(t ; . .).\right), s_{j}\left(\tilde{q}_{k}(t ; . .).\right), 0 \leq j \leq N-1, N \geq 1$, by the same inductive trick used above we achieve the boundedness of the sum of the derivatives of total height ${ }^{13} N$; this proves that also $s_{N}\left(\tilde{q}_{k}(t ; ., .).\right)$ and $s_{N}\left(\tilde{q}_{k}(t ; ., .).\right)$ are uniformly bounded.

Proposition 4.2 The symbol $q(t ; y, \xi)$, $\mathbf{S G}$ diffeomorphism with $\mathbf{S G}^{0}$ parameter dependence ${ }^{14}$, admits an inverse $\bar{q}(t ; x, \xi) \in \mathbf{S G}_{l}^{e_{2}}$ depending smoothly on $t$. $\bar{q}(t ; x, \xi)$ is defined on a closed subinterval of $J$ including 0 , which we continue denoting by $y^{15} J^{\prime}$. Moreover, $\bar{q}(t ; x, \xi) \in C^{\infty}\left(J^{\prime}, \mathbf{S G}_{l, c l}^{e_{2}}\right)$.

Proof. The first part of the Proposition is known ${ }^{16}$. With notations coherent with those used in Propositions 3.3 and 4.1, and an analogous calculation, we see that the condition

$$
\forall t \in J^{\prime} \forall(x, \xi) \in \mathbb{R}^{n} \times \mathbb{R}^{n} q(t ; \bar{q}(t ; x, \xi), \xi)=x,
$$

which characterizes $\bar{q}(t ; x, \xi)$, can be translated through $D^{e_{2}}$ as

$$
\begin{aligned}
\forall t \in & J^{\prime} \forall(y, \eta) \in B^{n} \times B^{n} \\
& \frac{1-[y]}{1-\left[\chi^{-1}\left(\frac{\tilde{\bar{q}}(t ; y, \eta)}{1-[y]}\right)\right]} \tilde{q}\left(t ; \chi^{-1}\left(\frac{\tilde{q}(t ; y, \eta)}{1-[y]}\right), \eta\right)=\chi(y)(1-[y]) .(4)
\end{aligned}
$$

Note that, setting

$$
f(t ; z, y, \eta)=\frac{1-[y]}{1-\left[\chi^{-1}\left(\frac{z}{1-[y]}\right)\right]} \tilde{q}\left(t ; \chi^{-1}\left(\frac{z}{1-[y]}\right), \eta\right)
$$

we have

$$
f(0 ; z, y, \eta)=\frac{1-[y]}{1-\left[\chi^{-1}\left(\frac{z}{1-[y]}\right)\right]}\left(1-\left[\chi^{-1}\left(\frac{z}{1-[y]}\right)\right]\right)
$$

\footnotetext{
${ }^{13}$ The only difference is that now the constants which appear in the estimates are given in terms of the $\tilde{a}_{j}, j=0, \ldots, N$ and $s_{j}\left(\tilde{q}_{k}(t ; .,).\right), s_{j}\left(\tilde{q}_{k}(t ; ., .).\right), j=0, \ldots, N-1$.

${ }^{14}$ See [5], Proposition 4.13 and Theorem 4.14.

${ }^{15}$ Actually, the present $J^{\prime}$ can be strictly included in the $J^{\prime}$ on which $q(t ; y, \xi)$ is defined.

${ }^{16}$ The proof can be found in [5] or [6].
} 


$$
\begin{gathered}
\cdot q\left(0 ; \frac{z}{1-[y]}, \chi(\eta)\right)=z \Rightarrow \\
\Rightarrow \quad \frac{\partial f}{\partial z}(0, z, y, \eta)=\operatorname{diag}(1, \ldots, 1) .
\end{gathered}
$$

Then, the equation

$$
f(t ; z, y, \eta)=\chi(y)(1-[y])
$$

can be solved with respect to $z$ in a suitably small closed interval centered in the origin, with arbitrary $(y, \eta)$. The resulting function clearly has the desired regularity up to the boundary, by the implicit function theorem and the fact that all the objects involved are in $C^{\infty}\left(J^{\prime}, C^{\infty}\left(B^{n} \times B^{n}\right)\right)$. Since there exists a function $\tilde{\bar{q}}(t ; ., ..) \in C^{\infty}\left(J^{\prime}, C^{\infty}\left(B^{n} \times B^{n}\right)\right)$ which satisfies (4.14), we have $\bar{q}(t ; ., .)=.\left(\left(D^{e_{2}}\right)^{-1} \tilde{\bar{q}}\right)(t ; ., .$.$) . This completes the proof.$

Proof of Theorem 1.2. After our preparation, the proof of Theorem 1.2 follows the same lines of the analogous Theorem 4.14 of [5]. We can set

$$
\begin{aligned}
\psi(t ; y, \xi) & =\langle y \mid \xi\rangle+\int_{0}^{t}\left[\lambda_{e}(s ; q(s ; y, \xi), p(s ; y, \xi))+\right. \\
& \left.-\left\langle\left(\nabla_{\xi} \lambda_{e}\right)(s ; q(s ; y, \xi), p(s ; y, \xi)) \mid p(s ; y, \xi)\right\rangle\right] d s
\end{aligned}
$$

and also

$$
\varphi(t ; x, \xi)=\psi(t ; \bar{q}(t ; x, \xi), \xi) \Leftrightarrow \varphi(t ; q(t ; y, \xi), \xi)=\psi(t ; y, \xi)
$$

As is well known, the function $\varphi$ defined in (4.20) is the solution of (4.2), and, as such, is a regular phase function. Moreover, using the results obtained in Propositions $3.3,4.1$ and 4.2 , it turns out that, indeed, $\varphi \in \mathcal{P}_{c l}^{\epsilon}$.

The amplitude $a(t ; x, \xi)$ can be obtained solving the so-called transport equations, each one giving a term $a^{(j)}$ of its asymptotic expansion ${ }^{17}$. Since $a^{(j)} \in C^{\infty}\left(J^{\prime} ; \mathbf{S G}_{l}^{-j e}\right)$, by Theorem 2.13, it is enough to prove $\forall j \in \mathbb{N} \quad a^{(j)} \in$ $C^{\infty}\left(J^{\prime} ; \mathbf{S G}_{l, c l}^{-j e}\right)$. We give the details of the proof of this fact for $a^{(0)}$. This term must satisfy the equation

$$
\left\{\begin{array}{l}
\left(D_{t}-\lambda_{0}\right) a^{(0)}-\sum_{|\alpha|=1} \partial_{\xi}^{\alpha} \lambda_{e} D_{\alpha}^{x} a^{(0)}-\left(\sum_{|\alpha|=2} \frac{\partial_{\xi}^{\alpha} \lambda_{e}}{\alpha !} \tilde{\sigma}_{\alpha}\right) a^{(0)}=0 \\
a^{(0)}(0 ; .)=1
\end{array}\right.
$$

with suitable ${ }^{18} \tilde{\sigma}_{\alpha} \in C^{\infty}\left(J^{\prime} ; \mathbf{S G}_{l}^{e_{1}-e_{2}}\right)$. Indeed, since $\tilde{\sigma}_{\alpha}$ only depends polynomially on the derivatives of $\varphi$, we have $\tilde{\sigma}_{\alpha} \in C^{\infty}\left(J^{\prime} ; \mathbf{S G}_{l, c l}^{e_{1}-e_{2}}\right)$. By the change of variables

$$
a_{\star}^{(0)}(t ; y, \xi)=a^{(0)}(t ; q(t ; y, \xi), \xi)
$$

\footnotetext{
${ }^{17}$ See again, e.g., [5] for the $\mathbf{S G}$ case of this well known construction. ${ }^{18}$ See again [5].
} 
we are reduced to the equation

$$
\left\{\begin{array}{l}
\frac{d}{d t} a_{\star}^{(0)}-i H a_{\star}^{(0)}=0 \\
a_{\star}^{(0)}(0 ; .)=1
\end{array}\right.
$$

with $H=\lambda_{0}+\sum_{|\alpha|=2} \frac{\partial_{\xi}^{\alpha} \lambda_{e}}{\alpha !} \tilde{\sigma}_{\alpha} \in C^{\infty}\left(J^{\prime}, \mathbf{S G}_{l, c \mathrm{l}}^{0}\right)$. The solution of (4.23) is

$$
a_{\star}^{(0)}(t ; y, \xi)=e^{i \int_{0}^{t} H(\tau ; y, \xi) d \tau} .
$$

Now, $H \in C^{\infty}\left(J^{\prime}, \mathbf{S G}_{l, c \mathrm{l}}^{0}\right)$ implies $\tilde{H}(t ; z, \zeta)=\left(D^{0} H\right)(t ; z, \zeta) \in C^{\infty}\left(J^{\prime}, C^{\infty}\left(B^{n}\right.\right.$ $\left.\times B^{n}\right)$ ) and also, obviously,

$$
\exp \left(i \int_{0}^{t} \tilde{H}(\tau ; z, \zeta) d \tau\right) \in C^{\infty}\left(J^{\prime}, C^{\infty}\left(B^{n} \times B^{n}\right)\right) .
$$

Again by Propositions 3.3, 4.1 and 4.2, we obtain $a^{(0)} \in C^{\infty}\left(J^{\prime}, \mathbf{S G}_{l, \mathrm{Cl}}^{0}\right)$, as desired. The argument can be repeated with almost no change for all the other terms $a^{(j)}, j \geq 1$, and this allows us to conclude.

\section{Acknoledgements}

Thanks are due to Prof. B.-W. Schulze, Prof. E. Schrohe and Dr. J. Seiler, Institute for Mathematics, University of Potsdam, and to Dr. D. Kapanadze, Mathematical Institute of the Academy of Sciences of Georgia (Tbilisi), for many helpful hints and discussions while the former of the authors was in Clausthal and in Potsdam, where part of this work was completed.

\section{References}

[1] P. Boggiatto, E. Buzano, and L. Rodino. Global Hypoellipticity and Spectral Theory. Akademie Verlag, Berlin, 1996.

[2] H. O. Cordes. The Technique of Pseudodifferential Operators. Cambridge Univ. Press, 1995.

[3] H. O. Cordes and A. K. Erkip. The $N$-th Order Elliptic Boundary Value Problem for Noncompact Boundaries. Rocky Mountain Math. J., 10:7-24, 1980.

[4] S. Coriasco. Fourier Integral Operators in SG classes I. Composition Theorems and Action on SG Sobolev Spaces. 1998. To appear in Rend. Sem. Mat. Univ. Pol. Torino.

[5] S. Coriasco. Fourier Integral Operators in SG classes II. Application to SG Hyperbolic Cauchy Problems. Ann. Univ. Ferrara, 47:81-122, 1998. 
[6] S. Coriasco. Fourier Integral Operators in SG classes with Applications to Hyperbolic Cauchy Problems. PhD thesis, Università di Torino, 1998.

[7] S. Coriasco and L. Rodino. Cauchy problem for SG-hyperbolic equations with constant multiplicities. Ric. di Matematica, 48, (Suppl.):25-43, 1999.

[8] Y. Egorov and B.-W. Schulze. Pseudo-Differential Operators, Singularities, Applications. Birkhäuser, 1997.

[9] L. Maniccia and P. Panarese. Eigenvalues Asymptotics for a Class of Elliptic Pseudodifferential Operators on Manifolds with Cylindrical Ends. 1999. To appear.

[10] A. Mohamed. Etude Spectrale des Operateurs Pseudodifferentiels Hypoelliptiques. $\mathrm{PhD}$ thesis, Institut de Mathématiques et d'Informatiques de l'Université de Nantes, 1983.

[11] E. Schrohe. Spaces of Weighted Symbols and Weighted Sobolev Spaces on Manifolds. In H. O. Cordes, B. Gramsch, and H. Widom, editors, Proceedings, Oberwolfach, number 1256 in Springer LNM, New York, pages $360-377,1986$.

[12] E. Schrohe. Complex Powers on Noncompact Manifolds and Manifolds with Singularities. Math. Ann., 281:393-409, 1988.

[13] E. Schrohe and A. K. Erkip. Normal Solvability of Elliptic Boundary Value Problems on Asymptotically Flat Manifolds. J. Funct. Anal., 109,1:22-51, 1992.

[14] B.-W. Schulze. Boundary Value Problems and Singular Pseudo-differential Operators. J. Wiley \& sons, Chichester, 1998.

[15] I. Witt. A Calculus for Classical Pseudo-Differential Operators with NonSmooth Symbols. Math. Nachr., 194:239-284, 1998. 